\title{
Estado nutricional y evolución postoperatoria en niños sometidos a cirugía mayor electiva
}

\author{
Josefina Matthias C. '; Carlos Castillo D. '; Verónica Marín B. '; Francisco Ossandón C. ${ }^{2}$
}

\begin{abstract}
Resumen
Fropjsito: descrioir el esiado ce nutrición en niños acmitidos sora cirugía mayor electiva y el efecto de la deficiercias de la nutición sovre la evo ución postoperator a inmediara. Ambito y Diseńo: esludio descriptivo en 81 niños de 2 meses a 15 años de ecad de sivuación socia y ezonćmica med'a y baja semetides a cirugia electiva, icrácica o abdominal, de más de 90 min de duracién con resección de visceras o manipulación prolongada de ellas. - lc largo de un año, en un hospital general públ'co asociado a ura escuelc ce medicina. Medicianes: se comparó el esado de nutricion entre unc y tres días anes de la inlervención y $z$ a $\&$ dics después de ésta, mediante peso para edad peso para talla, ral a para ejad $\left(\mathrm{P} / \mathrm{E}, \mathrm{P} / \mathrm{T}, \mathrm{T} / \mathrm{E}_{1}\right.$, área muscular braquiol, concentiación sérica de albimina, preabimina y zine y recuento de lintocitos. Resuritcolos: a ingresor al estudio, $18,5 \%$ de los pacientes lenian déficit nut:icional por $\mathrm{P} / \mathrm{E}<<90 \%$ en los menores te un añof o $\mathrm{P} / \mathrm{T}:<90 \%$ en los mayores de un eñol: $28,1 \%$ len an . $/ \mathrm{E}$ <95\%: en 33,3\% el árec ruscular braquial es aba bajo el percentil 15: er $2,5 \%$ la concentraciśn plasmácica de albumina era menos de $3,5 \mathrm{~g} / \mathrm{dl}$; en $10.9 \%$ la prealbimina pasmálica era igual o menor que $10 \mathrm{mg} / \mathrm{dl} ; 5 \%$ enían menos de 1500 infocios $/ \mathrm{ml}$ de sangre y en $25.4 \%$ to concentración sérico de zunc era menor que $100 \mathrm{\mu g} / \mathrm{dl}$ los porcenlojes de desnutrición -en las evaluaciones preoperatoria y postoperctoria- fueron, entre los menores de 24 meses $1 \% \mathrm{P} / \mathrm{E}$ O \% F/T) $28,9 \%$ y $44,0 \%$ ( $\mathrm{p}=0,027$, eri los niños de 2 a 5 años $10 \%$ y $20 \% 1 \% \mathrm{P} / \mathrm{Ti}$ ( $\mathrm{P}=0,0481$ \% en los de más de 5 años $8.0 \%$ y $8.0 \%$ (\% P/T) resoectivamente. La prevalencia de a teracionos aumentó después de la operación para todos os indicedores, excepc T/E y zinc plastrárico. Sólo el tipo de cirugía se asoció can el riesco de complicaciones postoperatorias. Conclussón: la prevalencia de desnutrición en rifios que requieren cirugía mayor es más allo que en la población genercl.
\end{abstract}

|Palabras clave: cirugio, estedo nutricicnal. complicaciones postoperatorios, intancia, niñez.]

\section{Prevalence of undernutrition and surgical complications in infants and children undergoing major elective surgery}

Objective: 10 describe nutritional slate in children a need of mopor elective surgery cnd to re ale it to ecrly surgical complications. Design: descriptive case series. Sething: public university ossocioned general hospita caring or micidle and lowie sorioeconom c status patienls at Santiago, Chile. Patients: all peciaric patients admitted for mojor elective obdominal or thoraxic surgery from morch 1992 throughout aprl 1993 . excepting those previously managed with paren'eral nutrition to: severi or more doys or those with severe underlying diseases itype l ciaberes mellitus, complex congenital cordiovoscular maltormatiors. hepatic or renal falure: Methods; weight for oge : $W / A$ and height $W / H$, height for age $\left[{ }_{1} / \mathrm{A}\right]$, arm muscle arec, serum albumir, prealbumin, blocd lymprocyte count and plasma zinc were prospective:y meassu'ed al adrission one 2 to $B$ days after operation to 81 parients aged nuo montrs to 15 years. Results: nutritional deficiency was ecorded ol acrission ir $18.5 \%$ patients by $\mathrm{W} / \mathrm{A}$ or $\mathrm{W} / \mathrm{H}$, in $28.1 \%$ oy $\mathrm{H} / \mathrm{A}$, in $33.3 \%$ by arm muscle area, in $2.5 \%$ by serum albumin ir $1.0 \%$ by preolbumin, in $5 \%$ by lymphocyte cout and in $25.4 \%$ by jerum zinc. Coriciusions: infarls were more offecled han presctool and school children $128,9 \% 10 \%$ and $0.6 \%$ respectivelyl. A significani ncrecse in jil criterig for nutritional deficit excegt $\mathrm{H} / \mathrm{A}$ and serum zinc wos recorded chter surgery. There was no significant association betweer -ut tional criteria and inciderce of surgical complicalions uthich were rather related to complexily of surgicel procedures. Prevalence of mal-utrition is higier among children ot need of eleckive mojor surgery thar, in the general pooulation, particularily at infoncy

(Key words: nutriticnal stalus, inta-1s, childen. surgery, operodive, pos'operative compl cations.]

1. Instiluto de Nutrición y Tecnología de los Alimentos (INTA), Universidad de Chile.

2. Antes: Servicio de Cirugía Infantil. Hospital San Juan de Dios. Actualmente: Servicio de Cirugia, Hospital de Niños Luis Calvo Mackenna.

Financiado parcialmente por: Beca PG-037-93, Departamento de Postgrado y Postítulo, como parte del programa de Magíster en Ciencias Médicas, Mención Nutrición, Faculrad de Medicina, Universidad de Chile. 
La prevalencia de desnutrición en pacientes hospitalizados es alrededor de 30 a $50 \%$, muy alta en comparación con lo que ocurre en la población general de adultos y niños ${ }^{1.9}$. Este déficit nutricional es de especial importancia en cirugía, pues se le asocia con más riesgo de complicaciones postoperatorias e incluso muerte en pacientes ${ }^{10-13}$. Desde el empleo de alimención parenteral total, a contar de 1968, se han iscado indicadores preoperatorios del estado $\geq$ nutrición que identifiquen a los grupos con ayor riesgo de complicaciones postoperatorias ara controlarlos oportunamente, y se han desaollado ecuaciones predictoras de complicacio*s postquirúrgicas en pacientes adultos como . de Mullen, basada en criterios nutricionales tropométricos y bioquímicos ${ }^{14}$. La prevalena de desnutrición en cirugía infantil fluctúa itre 20 y $40 \% 6,7,9,15$, pero la evolucion nutrional intrahospitalaria de estos pacientes y su ;ociación con complicaciones postquirúrgicas 3 ha sido descrita. Con los propósitos de estalecer la prevalencia de desnutrición al ingresar hospital y durante su hospitalización. así mo determinar si existe alguna asociacion con J evolución clínica, en niños que deben ser sotetidos a procedimientos quirúrgicos mayores ectivos, se evaluó el estado de nutrición en un rupo de ellos empleando criterios antropoméicos y bioquímicos, al ingreso y durante el peodo postoperatorio.

\section{Pacientes y Métodos}

Se incorporaron prospectivamente al estudia todos los ucientes aceptados en el Servicio de Cirugía Infantil del ospital San Juan de Dios, del Servicio de Salud Metropoano Occidente de Santiago, para cirugia mayor torácica o sdominal electiva, entre marzo de 1992 y absl de 1993. Is edades fluctuaton entre los 2 meses y los 15 anos. Se sfinió como cirugía mayor la de torax o abdornen que cluía resección de vísceras o manipulación prolongada z ellas por un tiempo operatorio mayor a 90 min y menor 180 min y gran cirugia los procedimientos torácicos, absminales o toracoabdominales con resección, manipulaon, movitización o modificación de vísceras por tiempo yesatorio igual o mayor a 180 minutos ${ }^{16}$. Se excluyeron il análisis los pacientes que: recibieron apoyo nutricional arenteral mayor a siete días antes de la operacion; sufrían Ifennedades graves asociadas a afecciones geneticas, diates mellitus dependiente de insulina, malformaciones rdiovasculares congénitas complejas, falla hepática o real; habian recibido iratamiento corticoesteroidal o innuosupresor; habían sido sometidos a ciruzía en los tres úl- timos meses antes de ingresar; eran menores de un año con antecedentes de prematurez.

En el periodo preoperatorio inmediato 11 a 3 días antes de la intervención) y en el postoperatorio ( 2 a 8 días después de ella) se determinaron peso, talla, perimetro braquial (PB), pliegue tricipital (PT), peso/edad (P/E), pesof calla (P/T), talla/edad (T/E), area muscular braquial (AMB) para cada paciente. Los estándares para $P / E, P / T$ y T/E fueron los de OMS-NCHS ${ }^{13}$. La información obtenida se expres $\sigma$ como porcentaje del estándar. Para definir déficit nutricional se usó la relación P/E en los menores de un año y $\mathrm{P} f \mathrm{~T}$ en los mayores de un año ${ }^{18}$. Los estándares para PT, $\mathrm{PB}$ y AMB tuerou los de Frisancho ${ }^{14}, 20$, agtupándose los datos como número de niños bajo determinados percentiles. Como indicadores bioquímicos se estudiaron albúmina plasmática (por técnica de verde de bromocresol), prealbúmina plasmatica (por tecnica de nefelometría), recuento de linfocitos (obtenido del hemograma) y zinc plasmático (por técnica de espectrofotometrúa de absorción atónica). Se determinó también hematocrito, hemoglobina, recuento de leucocitos y la velocidad de sedimentación de los eritrocitos VHS (en los mayores de 12 meses).

Se definió deficit nutricional por uno o más de los siguientes indicadores: relación P/T $<90 \%$ en los mayores de un año y P/E $<90 \%$ en los menores de un año, T/E

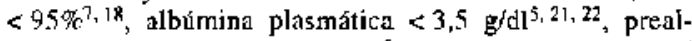
búmina plasmática $\left.<10 \mathrm{mg} / \mathrm{dl}^{5}\right)$, área muscular braquial $<\mathrm{p} 10$ en los mayores de un año $y<\mathrm{p} 15$ en los menores de un ano ${ }^{15,21}$, recuento de linfocitos $<1500 \mathrm{cel} / \mathrm{ml}$ en los mayores de 6 meses $y<2500 \mathrm{cel} / \mathrm{ml}$ en los menores de 6 meses ${ }^{2,15,21}$ y zitac plasmático $<100 \mathrm{ug} / \mathrm{dl}^{23}$.

Las complicaciones postoperatorias se clasificaron como muerte, complicaciones mayores, complicaciones menores y sin complicaciones ocuridas en un período no mayor a 14 días posterior al acto quirúrgico o hasta el alta del paciente. De las complicaciones se definieron: sepsis (dos hemnocultivos positivos, fiebre, astenia y adinamia $y$ eventualmente hipotensión e hipoperfusión); absceso intraabdominal (fiebre, imágenes sugerentes y necesidad de dren@je quirúrgico); neumonía (fjebre, polipnea, tos e imagenes radiográficas compacibles); dehiscencia de la herida operatoria (separación de los labios de la herida que requiere cierre quirúrgico); evisceracion (separación de todos los planos operatorios que requiere cierre quirúrgico); infeccion de la berida operatoria (signos inflamatorios locales, cultivo positivo de la herida operatoria positivo, que requiere drenaje).

Se registró la alimentación postopemtoria del paciente en los siete días siguientes al procedimiento quirúrgico y se agrupó en cuatro tipos: ayuno (régimen cero por 405 días $y$ luego régimen hídrico o líquido por 2 a 3 días): semiayudo (régimen hídrico o lfquido por 4 a 5 días y luego régimen liviano por 2 a 3 días); alimentación oral habitual (régimen común recibido en más del $80 \%$ de su indicación - alimentación enteral desde el primer o segundo día y hasta por lo menos el séptimo día postoperatorio); nutrición parenteral (desde el primero o segundo día, hasta por Io menos el séptimo día postoperatorio\}.

Se usó paquete estadistiço S.A.S. (Statistical Analysis System). Para el anúlisis de asociación entre variables seleccionadas se utilizó estadística chi cuadrado, t de Student y rest de Fisher con un aivel de confianza del 9.5\%. Para estudiar si existe alguna capacidad predictiva de riesgo de 
complicaciones posterior a la cirugía en las variables tanto antropométricas como bioquímicas estudiadas en el periodo preoperatorio, se usó análisis de regresión logistica

\section{Resultados}

Se estudiaron 81 pacientes, 50 mujeres $(61,7 \%)$ y 31 hombres $(38,3 \%)$, de los cuales 38 (47\%) eran lactantes, $20(25 \%)$ preescolares y $23(28 \%)$ escolares. El tipo de cirugía fue cirugía mayor en 69 casos $(85,2 \%)$ y en 12 gran cirugía $(14,8 \%)$. La cirugía realizada fue de tipo cardiopulmonar y tímica en 25 casos $(30,8 \%)$, gastrointestinal y hepatobiliar en $25(30,8 \%)$ y urogenital y renal en $31(38,2 \%$ ) (tabla 1 ).

La prevalencia de desnutrición en los menores de 6 años de esta muestra fue 4,5 veces mayor por $\mathrm{P} / \mathrm{E}(42,6 \%$ ante $9,4 \%)$ y 8 veces mayor por $\mathrm{P} / \mathrm{T}(21,3 \%$ ante $2,6 \%)$ que entre la población coetánea atendida en la misma área geográfica (Servicio de Salud Metropolitano Occidente) durante el año 1992 (tabla 2).

La prevalencia de desnutrición en el preoperatorio según porcentaje $\mathrm{P} / \mathrm{E}$ en los menores de 1 año y $P / T$ en los mayores de 1 año, fue de $6,2 \%$ de desnutrición severa, $3,7 \%$ moderada y $8,6 \%$ leve, sin aumento significativo postoperatorio. En el preoperatorio $28,9 \%$ de los lactantes, $10 \%$ de los preescolares y $8,6 \%$ de los escolares tenían desnutrición mientras en el postoperatorio las proporciones correspondientes fueron $44,6 \%, 20 \%$ y $8,6 \%$ respectivamente, siendo significativas las diferencias de antes a después de la operación para lactantes $(p=0,027)$ $y$ preescolares $(p=0,048)$.

La prevalencia de compromiso nutricional pre y postoperatorio según los indicadores antropométricos y bioquímicos estudiados y como para todos ellos aumentó después de la operación (tabla 2). Las diferencias son significativas en todos los indicadores ( $t$ de Student para muestras pareadas) excepto en zinc plasmático y talla para edad.

La alimentación postoperatoria de estos pacientes consistio en ayuno en 10 , semiayuno en seis, alimentación oral habitual en 53 y nutrición parenteral en 12 casos. No se encontró asociación significativa (prueba de Fisher) entre ella y las complicaciones postoperatorias. Dieciséis niños sufrieron 17 complicaciones postoperatorias $(20,9 \%)$ : seis mayores (sepsis, bronco-

\section{Tabla 1}

Motivo de la cirugfa mayor electiva en 81 pacientes pediátricos

Diagnóstico postoperatorio

Frecuencia

Cardiopulmonar y tímico

Ductus arterioso persistente

Secuestro pulmonar y hernia diafragmatica

Miastenia gravis

23

125

\section{Gastrointestinal $y$ hepatobiliar}

Enfermedad de Hirschprung

Hernia diafragmática

Malformación ano-rectal

Cavernomatosis de la porta

Atresia de vias biliares

Atresia de esófago

Acalasia de esófago

Reflujo gastroesofágico

Colelitiasis

Divertículo de Meckel

Otras

\section{Urogenital y renal}

Reflujo vésico-uretra]

Obstrucción pielo-ureteral

Ureterocele

Vejiga neurogénica

Tumor ovárico

Otras

neumonía, evisceración cubierta, obstrucción intestinal por bridas y dos dehiscencia de sutura) y 11 complicaciones menores (seis infecciones urinarias y cinco infecciones de herida operatoria). No se encontró asociación entre la variación del pre al postoperatorio de peso, $\% \mathrm{P} / \mathrm{E}$ o $\mathrm{P} / \mathrm{T}$, albúmina, prealbúmina o zinc plas-mático y complicaciones postoperatorias.

El análisis de regresión logística para identificar factores predictores de complicaciones postoperatorias entre las variables edad, sexo, diagnóstico postquirúrgico, tipo de cirugía, procedencia, \% P/E o P/T preoperatoria, AMB preoperatoria, albúmina plasmática preoperatoria. 
Tabla 2

Prevalencia de desnutrición pre y postoperatoria según indicadores antropométricos y bioquímicos

\begin{tabular}{|c|c|c|c|c|c|c|}
\hline \multirow{2}{*}{ Indicador nutricional } & \multicolumn{3}{|c|}{ Preoperatorio } & \multicolumn{3}{|c|}{ Postoperatorio } \\
\hline & $\mathbf{n}$ & frecuencir & $\%$ & $\mathbf{n}$ & frecuencia & $\%$ \\
\hline$\%$ P/E o P/T $<90 \% * * *$ & 81 & 15 & 18,5 & B I & 23 & 28,4 \\
\hline$\% \mathrm{~T} / \mathrm{E}<95 \%$ & 78 & 22 & 28,1 & 78 & 22 & 28,1 \\
\hline PT $<p \mid 100$ IS**:k & 81 & 8 & 9,8 & $8 t$ & 18 & 22, ] \\
\hline$P B<p \mid 0015^{* A *}$ & 81 & 27 & $3,3.3$ & $B I$ & 36 & 44,4 \\
\hline $\mathrm{AMB}<p 10015^{*}$ & 81 & 27 & 33,3 & $8 \mathrm{~L}$ & 30 & 37.5 \\
\hline Alb, pl $<3.5 \mathrm{~g} / \mathrm{dl}]_{* *}^{*}$ & 81 & 2 & 2,5 & 81 & 7 & 8.6 \\
\hline 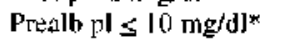 & 57 & 6 & 10.9 & 52 & 10 & 19,2 \\
\hline Linf $<1500 / \mathrm{ml}^{*}$ & 79 & 4 & 5,0 & 80 & 5 & 6.2 \\
\hline Zint $\mathrm{pl}<100 \mu \mathrm{g} / \mathrm{dl}$ & 63 & 16 & 25.4 & 57 & 22 & 38.5 \\
\hline
\end{tabular}

PT: pliegue tricipital : PB: perínetro braquial: AMB: áea muscular braquiaj; Alb: albúminá plasmática: PA: prealbúmina plasmatica; Linf: linfocilos sangre: pi: plasmático.

T par Student, * $\mathrm{p}<0,025, *{ }_{\mathrm{p}}<0,0025 .{ }^{* * *} \mathrm{p}<0.001$

recuentos de linfocitos preoperatorios, hematocrito preoperatorio, hemoglobina preoperatoria, tiempo operatorio y cirujano (las demás variables no se consideraron por datos incompletos), otorgó valor predictor sólo al tipo de cirugía $(p=0,0015)$.

\section{Comentario}

Se han descrito prevalencias de hasta $50 \%$ de desnutrición calórico-proteínica aguda en pacientes adultos hospitalizados ${ }^{2}$ y de 36 a $39 \%$ en niños hospitalizados para cirugía electiva en Estados Unidos 9.15 . En Chile se describio en $1981^{7}$ prácticamente el doble de prevalencia de desnutrición en un grupo de pacientes pediátricos quirúrgicos en general empleando criterios de $\% \mathrm{P} / \mathrm{E}, \% \mathrm{P} / \mathrm{T}$ y $\%$ T/E pero ambos resultados en realidad no son comparables porque los recién mencionados fueron obtenidos en 1978 de un corte transversal e incluyeron pacientes de cirugía electiva y de urgencia y ha habido cambios objetivos en la prevalencia de desnutrición entre los niños chilenos desde entonces. Si bien no podemos concluir que la desnutrición ha disminuido en los niños que ingresan al hospital para cirugía, la proporción de desnutridos encontrada en nuestro estudio son más bajos que los descritos previamente en niños y adultos, lo que podría reflejar cierta tendencia positiva.

La mayor prevalencia de desnutrición en este tipo de pacientes que en la generalidad de los atendidos en el mismo servicio de salud donde se efectuó la investigación puede explicarse por el tipo de afecciones de base de los pacientes seleccionados, que suele afectar la nutrición por dificultar la alimentación, reducir la eficiencia energética, aumentar el gasto energético o provocar pérdidas anormales de nutrientes ${ }^{24}$.

La deficiencia nutricional de los pacientes de esta serie fue preferentemente marásmica, con déficit de tejido graso y proteína muscular y poco deterioro en las proteínas viscerales e inmunitario. La mayor prevalencia de desnutrición al Ingreso en los lactantes, refleja su mayor vulnerabilidad y la seriedad de la enfermedad de base, que demanda cirugía mayor tan temprano, sin permitir espera hasta una edad más segura o la corrección de su estado de nutrición ${ }^{25}$. Igualmente tanto ellos como los preescolares tienen más riesgo de sufrir deterioro nutricional por efecto de las condiciones prevalentes en el medio intrahospitalario, pues prácticamente duplican la cifra de desnutrición leve en el período postoperatorio. Esto podría deberse a que, por su situación nutricional límite en el preopera- 
torio, frente al apremio de la cirugía y condiciones intrahospitalarias se desencadene una desnutrición leve ${ }^{26.27}$. También es posible suponer que la respuesta al estrés quirúrgico sea distinta en lactantes y preescolares, pero esta hipótesis ha sido poco estudiada.

La albúmina y la prealbúmina plasmáticas, que han sido frecuentemente utilizadas para evaluar el estado y la respuesta al apoyo nutricionales, parecen más bien variar en estos pacientes como parte de la reacción metabólica al estrés y no necesariamente por deterioro de la nutrición, pues ambas disminuyen en menos de $24 \mathrm{~h}$ desde el comienzo de un apremio como la cirugía, y pueden ser del orden de $25 \%$ en la albúmina y $50 \%$ en la prealbúmina, con respecto a las concentraciones normales ${ }^{28}$.

La falta de asociación entre estado nutricional preoperatorio y complicaciones postoperatorias no corresponde a lo esperado según la información disponible en adultos $5.14,21.79-34,10$ que sugiere que enfermos con déficit menos severos no requeririan intervención nutricional intensiva preoperatoria. Pocos de nuestros pacientes mostraban déficit severo de la nutrición (con afectación de todos a casi todos los indicadores empleados) y los que IJegaban en tales condiciones recibieron apoyo nutricional preoperatorio, generalmente parenteral, quedando excluidos de nuestro estudio.

\section{Referencias}

1. Willard MD. Gilsdorf RB, Price RA: Protein-calorie malnutrition in a community hospital. JAMA [980: 243; $1720-1722$

2. Bisthith BR. Blackbun GL. Hallowetl E. ef at: Protein status of general surgical patıents. JAMA I974; 23 : 8.58-860.

3. Bismiln BR, Blakbum $G L$, Virale J, er at: Prevalence of malnutrition in general medical patients. JAMA 1976: 235: 1567-1570.

4. Bumout $D$. Barrera $G$. Garras $V$, et at: Estado nutritivo de sujetos adultos hospitalizados: un estudio Iransversal. Rev Méd Chile 1980: 108: 700-706.

5. Pettigren RA. HilI GL: Indicators of surgical risk and clinical judgement. Br J Surg 1986; $73: 47.51$.

6. Parsm $H G$, Francoerr $T E$, Howland $P$, et at: The nutritional status of hospitalized children. An J Clin Nutr 1980; 33: 1140-1146.

7. Fucries A. Hertrampf $E$. Barrera $G$ : Evaluación tutricional del paciente pediátrico hospitalizado. Rev Chil Pediatr 1981: 52; 387-395.

B. Becerra M, bánez $S$, Castillo C: Evaluación nutricional en lactunies hospitalizados. Rev Chil Pediatr 1986: $57: 237-240$
9. Cooper A. Jakobouski D. Spiker J, et al: Nutritional assessment: an integral part of the preoperative pediatric surgical evaluation, J Pediatr Surg 198I; 16: $554-560$.

10. Weilkims $J$. Pietse'h JB, Buberick $O$, el af: Delayed hipersensitivity: indicator of acquired failure of host defenses in sepsis and trauma. Ann Surg 1976; 186: 241-249

11. Puetsoth JB, Meakins IL. Mac Lean LLD: The delayed hipetsensitivity response: applicarion in clinical surgery. Surgery $1977 ; 82 ; 349-355$.

12. Mullen $M L$, Germer $M L$. Buzby GP. er $a$ : inplications of Inalnutrition in the surgical parient. Arch Surg 1979; 114: 121-125.

13. Mullen JL: Consequences of mainutrition in the surgical patient. Surg Clin North Am 1981: 6I: 465. 487 .

14. Braby GP. Mulles JL, Mathews DC et at: Prognostic nutritional index in gastrointestinal strgery. Am J Surg 1980; 139: 160-167.

15. Merrit RJ and Suskind RH: Nutritional survey of hospitalized pediatric patients. Am J Clin Nutr 1979: 32: $1320-1325$.

16. Anand KJS, Aystey-Green 4: Measuring the severity of surgical stress in newborn infants. I Pediatr Surg 1988; $23: 297-305$.

17. NCHS Growth Curves for Children. Birth - 18 years. DHEW Publication $N^{\circ}$ (PHS)78-1650 Series lJ-N. 165.

13. Waterlow $J$ : Some aspects of childhood malnuirition as a public health problem. Brit Med J 1974: 4: 88.

19. Frisuncho AR: Triceps skin fold and upper arm muscle size norms for assessment of nutritional status. Am J Clin Nutr 1974: 27: 1052-1058.

20. Frisancho AR: New norms of upper limb fat and muscle areas for assessnent of nutritional status. Am J Clin : Vutr 1981: 34: 2540-2545.

21. Merrit RJ, Blachbum GL: Nutritional assessment and metabolic response to illiness of the hospitalized child En Suskind R.M. Textbook of Pediatric Nutrition. New York. Raven Press 1981: 28.5-307.

22. Dewipsey DT, Mullen JL, Buzby JP: The link between nutritional scatus and clinical outcome: can nutritiona] intervention nodify it?. Am J Clin Nutr 1988: 47:352. 356.

23. Orgill D and Demling RH: Current concepts and approaches to the wound healing. Crit Care Med 1988: 16: 899-908.

24. Uerry R, Gazitúa R: Evaluación del estado mutricional de] enfermo hospitalizado. Rev Med Chile 1980; 108: $542-550$

25. Mala $\omega$, Urrutia $J$, Aberasi $C$. ef $a l$ : Influence of recuirent infections on nutrition and growth of children in Guatemala. Am J Clin Nutr 1972; 25: 1267-1275.

26. Gattis Y. Fuentes A, Jarpa S, es ad: Situación alimentacia de pacientes pediátricos hospitalizados. Rev Chil Pediatr 1981; 52: 397-404.

27. Marin V. Castito C. Mashiuss $J$ : Nutrición en el enfermo quirúrgico pediátrico. Rev Chil Pediatr 1995; 66: $59-67$. 
28. Chwals WJ: Metabolism and nutritional frostiers in pediatric surgical patients. Pediatr Surg 1992; 72 : $1237-1266$.

29. Harvey KB, Moldawer LL. Bistrian BR, ef al: Biological measures for the formulation of a hospital prognostic index. Am J Clín Nutr 1981: 34: 2013-2022.

30. Yamanaka $H$, Nishi $M$, Kanenaki $T$, et al: Preoperative nutritional assessment to predict postoperative complication in gastric cancer patients. JPEN 1989; 13: 286-291.

31. Leite JF, Antunes CF, Monseiro JC, es al: Value of nutritional parameters in the prediction of postope- rarivo complications in elective gastrointestinal surgery. Br J Surg 1987; 74: 426-429.

32. Roy LB, Edwards PA, Barr LH: The value of nutritional assessment in surgical patient. JPEN 1985; 9 : 170-171.

33. Hirsch $S$, de Obaldia $M$. Petermann $M$. et al: Nutritional status of surgical patients and the relationship of nutritional to postoperative outcome. I Am Coll Nutr 1992: 11: $21-24$

34. Campos $A C$ and Meguid MM: Critical appraisal of the usefulness of perioperative nutritional support. Am J Clin Nutr 1992: 55: 117-130. 